\title{
SDA FULL-WAVE ANALYSIS OF BOXED MULTISTRIP LINES OF FINITE THICKNESS EMBEDDED IN
} A LAYERED LOSSY MEDIUM

\author{
A.Deleniv ${ }^{*}$, M. Gashinova ${ }^{* *}$, and I. B. Vendik ${ }^{* *}$ \\ * Chalmers University of Technology, Kemivägen 9, SE-412 96 Göteborg, Sweden, \\ Phone: +4631-772 3605, Fax:+4631-164513, Email:anatoli@ep.chalmers.se \\ ** Electrotechical University, St.Petersburg, 197376, Russia, Phone/Fax: +7(812)3460867, Email: mwlab@eltech.ru
}

\begin{abstract}
Spectral-domain approach (SDA) is applied to modeling propagation characteristics of multiconductor structure on superconducting strip lines with signal strips and ground planes of finite thickness in lossy layered media. Equivalent surface impedance is used to transform the superconducting strip of finite thickness into an infinitely thin strip. A novel procedure of a calculation of the equivalent surface impedance is proposed. Two sets of basis functions (Chebyshev and Legendre polynomials) are compared by their ability to accurate modeling of microwave losses in the structure. The model applicability is illustrated by numerical results compared with experimental and simulated data.
\end{abstract}

\section{THEORETICAL ANALYSIS}

A CAD tool for design of filters based on sections of multicoupled planar transmission lines Vendik et al. (1) is developed and improved. An efficient full-wave method is derived to calculate complex propagation parameters of the boxed multi-conductor planar lines embedded in a layered isotropic media as shown in Fig. 1. The method can be applied to the analysis of superconducting and normal metal lines.

The dielectric layers are characterized by the thickness $h_{U p}^{i}, h_{L o w}^{j}$ and the complex dielectric permittivity

$$
\varepsilon_{U p(\text { Low })}^{i(j)}=\varepsilon^{i(j)}-j \sigma^{i(j)} / \omega
$$

For a superconducting layer the dielectric constant is determined as

$$
\varepsilon_{U p(L o w)}^{i(j)}=-j \sigma_{s c}^{i(j)} / \omega
$$

where $\sigma_{s c}$ is the complex conductivity of a superconductor, Vendik (2).

The goal of the analysis is to define a vector of the complex propagation constants $k_{z}(\omega)=\beta(\omega)-j \alpha(\omega)$ in the wave propagation direction $z$ and the characteristic impedance for each mode propagating in the structure.

With the commonly used spectral domain notation, the linear relationships between fields and currents on the conductor interface can be written as

$$
\left[\begin{array}{c}
Z_{s}^{e q} \widetilde{J}_{z}\left(\alpha_{n}\right) \\
Z_{s}^{e q} \widetilde{J}_{x}\left(\alpha_{n}\right)
\end{array}\right]=\left[\begin{array}{cc}
\widetilde{Z}_{z z} & \widetilde{Z}_{z x} \\
\widetilde{Z}_{x z} & \widetilde{Z}_{x x}
\end{array}\right]\left[\begin{array}{c}
\widetilde{J}_{z}\left(\alpha_{n}\right) \\
\widetilde{J}_{x}\left(\alpha_{n}\right)
\end{array}\right],
$$

where $Z_{s}^{e q}$ is the equivalent surface impedance, $\widetilde{Z}_{i j}$ is the component of Green's dyad, $\widetilde{J}_{i, j}$ is the component of the surface current density, $\alpha_{n}$ is the Fourier variable. We use the analytical procedure for evaluation of the spectral Green's dyad based on so-called immitance approach Itoh (3). An extension to the lossy case is made by use of complex permittivity $\varepsilon^{i(j)}$ for $i$-th $\left(j\right.$-th) dielectric layer and the complex propagation constant $k_{z}$. Contribution of the superconducting strip conductivity to the loss factor can be accurately estimated with a modified set of boundary conditions applied to an infinitely thin strip. The equivalent surface impedance is used to transform a superconducting strip of finite thickness into an infinitely thin strip (Fig. 2). The real superconducting strip is characterized by the tangential electric and magnetic field components on the upper and lower interfaces related by impedance matrix of a thin conducting layer Van den Berghe et al.(4):

$$
\left[\begin{array}{c}
\widetilde{E}_{1} \\
\widetilde{E}_{2}
\end{array}\right]=\vec{n} \times\left[\begin{array}{cc}
1 & 0 \\
0 & -1
\end{array}\right]\left[\begin{array}{ll}
Z_{11} & Z_{12} \\
Z_{21} & Z_{22}
\end{array}\right]\left[\begin{array}{c}
\widetilde{H}_{1} \\
\widetilde{H}_{2}
\end{array}\right]
$$




$$
\begin{aligned}
& Z_{11}=Z_{22}=-j Z_{s c} \cot \left(\beta_{s c} t\right) \\
& Z_{12}=Z_{21}=j Z_{s c} \csc \left(\beta_{s c} t\right)
\end{aligned}
$$

where $Z_{\mathrm{sc}}$ is the surface impedance and $\beta_{\mathrm{sc}}$ is the complex propagation constant of the superconductor. We define the electric field in the plane $0 \leq y \leq t$ of the original strip as

$$
\widetilde{E}(y)=\frac{\sinh \left(\beta_{s c} y\right)}{\sinh \left(\beta_{s c} t\right)} \widetilde{E}_{1}-\frac{\sinh \left(\beta_{s c}(y-t)\right)}{\sinh \left(\beta_{s c} t\right)} \widetilde{E}_{2}
$$

and use the following approximate ratio of the magnetic fields on the upper and lower interfaces for lossless dielectric media

$$
\left|\frac{\widetilde{H}_{1}}{\widetilde{H}_{2}}\right|=\frac{\varepsilon_{U p}^{1}}{\varepsilon_{\text {Low }}^{1}} .
$$

Keeping the equality between the power stored and dissipated within the volume of a real superconducting strip and the equivalent infinitely thin one, the following equation for the eqivalent surface impedance of infinitely thin strip was obtained

$$
Z_{s}^{e q}=\frac{1}{\sigma_{s c}\left(1+\left(\varepsilon_{\text {Low }}^{1} / \varepsilon_{U p}^{1}\right)\right)^{2}} \int_{0}^{h}\left|\frac{\sinh \left(\beta_{s c} y\right) \sigma_{s c}}{\sinh \left(\beta_{s c} t\right)} k 1-\frac{\sinh \left(\beta_{s c}(y-t)\right) \sigma_{s c}}{\sinh \left(\beta_{s c} t\right)} k 2\right|^{2} d y,
$$

where

$$
k 1=Z_{11}-Z_{12} \frac{\varepsilon_{\text {Low }}^{1}}{\varepsilon_{U p}^{1}}, k 2=Z_{11} \frac{\varepsilon_{L o w}^{1}}{\varepsilon_{U p}^{1}}-Z_{12}
$$

In performing solution for planar transmission lines by SDA, we consider two different sets of basis functions $\varphi 1$ and $\varphi 2$ to expand the unknown electric currents of the system (3). The set $\varphi 1$ is the first type of Chebyshev polynomials $T_{p}$ with Maxwell weighting function. The set $\varphi 2$ is based on orthogonal Legendre polynomials $P_{p}$ and is suitable for modeling finite edge current. In our codes, we use these sets to model both the axial current density and the derivative of the transversal one

$$
J_{z}(x)=\sum_{k} \sum_{p} a_{p}^{k} \varphi_{p}^{k}(x), \quad J_{x}^{\prime}(x)=\sum_{k} \sum_{p} b_{p}^{k} \varphi_{p}^{k}(x) .
$$

The system of linear equations (3) has to be arranged to a homogeneous one. We formulate two different systems to be solved using the sets $\varphi 1$ and $\varphi 2$ :

$$
\left[\begin{array}{c}
\widetilde{E}_{z}\left(\alpha_{n}\right) \\
\widetilde{V}_{x}\left(\alpha_{n}\right)
\end{array}\right]=\left[\begin{array}{cc}
\widetilde{Z}_{z z}-Z_{s}^{e q} & \frac{\widetilde{Z}_{z x}}{-j \alpha_{n}} \\
\frac{\widetilde{Z}_{x z}}{-j \alpha_{n}} & \frac{\widetilde{Z}_{x x}-Z_{s}^{e q}}{-\alpha_{n}{ }^{2}}
\end{array}\right]\left[\begin{array}{c}
\widetilde{J}_{z}\left(\alpha_{n}\right) \\
\widetilde{J}_{x}{ }^{\prime}\left(\alpha_{n}\right)
\end{array}\right]
$$

for $\varphi 1$ and

$$
\left[\begin{array}{c}
\Pi \widetilde{E}_{z}\left(\alpha_{n}\right) \\
\widetilde{V}_{x}\left(\alpha_{n}\right)
\end{array}\right]=\left[\begin{array}{cc}
\frac{\widetilde{Z}_{z z}-Z_{s}^{e q}}{-\alpha_{n}^{2}} & \frac{\widetilde{Z}_{z x}}{j \alpha_{n}{ }^{3}} \\
\frac{\widetilde{Z}_{x z}}{-j \alpha_{n}} & \frac{\widetilde{Z}_{x x}-Z_{s}^{e q}}{-\alpha_{n}{ }^{2}}
\end{array}\right]\left[\begin{array}{c}
\widetilde{J}_{z}\left(\alpha_{n}\right) \\
\widetilde{J}_{x}{ }^{\prime}\left(\alpha_{n}\right)
\end{array}\right],
$$

for $\varphi 2$, with

$$
V_{x}(x)=\int_{-L}^{x} E_{z}\left(x^{\prime}\right) d x^{\prime},
$$




$$
\Pi E_{z}(x)=\int_{-L}^{x} \int_{-L}^{x} E_{z}\left(x^{\prime}\right) d x^{\prime} d x^{\prime} .
$$

The both systems can be presented in general form as

$$
\left[\widetilde{E}\left(\alpha_{n}\right)\right]=\left[\widetilde{F}\left(\alpha_{n}, \omega, k_{z}\right)\right]\left[\widetilde{J}\left(\alpha_{n}\right)\right]
$$

Solving (15) by Galerkin's method leads to a homogeneous system of linear equations

$$
\left[A\left(\omega, k_{z}\right)[X]=0\right.
$$

with $[\mathrm{X}]$ being unknown expansion coefficients of the current density. The entries of $\left[\mathrm{A}\left(\omega, \mathrm{k}_{\mathrm{z}}\right)\right]$ are numerical series , whose general term in (16) is

$$
A_{p, q, v}^{k, j, \mu}=\frac{1}{\sum_{n} \phi_{p}^{k}\left(\alpha_{n}\right)\left[\tilde{\varphi}_{p}^{k}\left(\alpha_{n}\right)\right] *} \sum_{n}{\varphi_{p}^{k}}_{p}\left(\alpha_{n}\right) \widetilde{F}_{\mu v}\left(\alpha_{n}, \omega, k_{z}\right)\left[\widetilde{\varphi}_{q}^{j}\right] *
$$

where $\mu, \nu$ stand for $\mathrm{x}$ - or $\mathrm{z}$ - components of spectral dyad, $\mathrm{k}, \mathrm{j}=1 . . \mathrm{n}$ are the strip numbers, $\mathrm{p}, \mathrm{q}$ are the numbers of basis function.

In order to reduce numerical efforts for solving the dispersion equation, the effective speed-up algorithm in the case of using the basis function set $\varphi 1$ was implemented Gano et al. (5), while for the set $\varphi 2$ the large argument approximation for Legendre polynomial basis functions was used.

\section{NUMERICAL RESULTS.}

The convergence behavior of Galerkin's method for both sets of basis function was studied. The propagation constants versus number of applied basis function are shown in Fig. 3 for superconducting (YBCO) microstrip line. Evidently, the Chebyshev polynomial-based set demonstrates quite poor convergence (typically, more than 15 basis functions have to be applied to provide $1 \%$ accuracy). It makes this basis set computationally inefficient. The basis set based on the Legendre polynomials shows better behavior (6-7 basis functions are enough to obtain the result close to converged value). All data presented below are computed using 7 basis functions of this set.

A comparison between results of calculation of the HTS multistrip structure characteristics by the method developed with the experimental characteristics was done for the multistrip structure of two coupled S-shape YBCO resonators on $\mathrm{LaAlO}_{3}$ substrates (Fig. 4). Simulated and measured transmission coefficients at two different temperatures $(\mathrm{T}=20 \mathrm{~K}$ and $77 \mathrm{~K}$ ) Vendik et al. (6) are presented in Fig. 5. A good agreement gives a reliable confirmation of correctness of the method developed.

\section{CONCLUSIONS}

A computationally efficient full-wave method of analysis of HTS multistrip multilayered structure is proposed and discussed. The method allows taking into account real characteristics of the dielectric layers and conducting strips of a finite thickness. Numerous examples of simulation of different structures revealed a high efficiency and reliability of the method. As result, the complex propagation constants and related modal impedances of the multiconductor structure containing superconducting (normal metal) strip and lossy multilayer dielectric can be calculated. The method can be effectively used for a simulation and design of planar filter structures including tunable devices using ferroelectric layer.

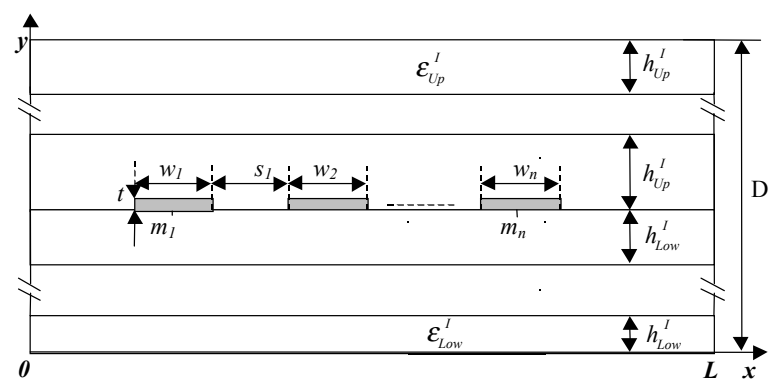

Fig. 1. Transverse section of multistrip structure in a layered media

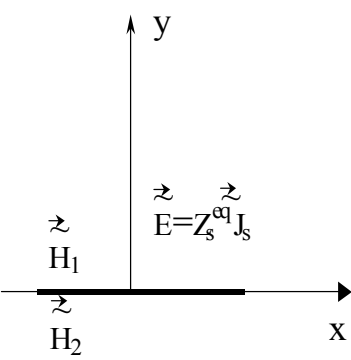

a)

Fig. 2. Equivalence between tangential boundary conditions for a strip of thickness $t$ (a) and infinitely thin equivalent (b). 


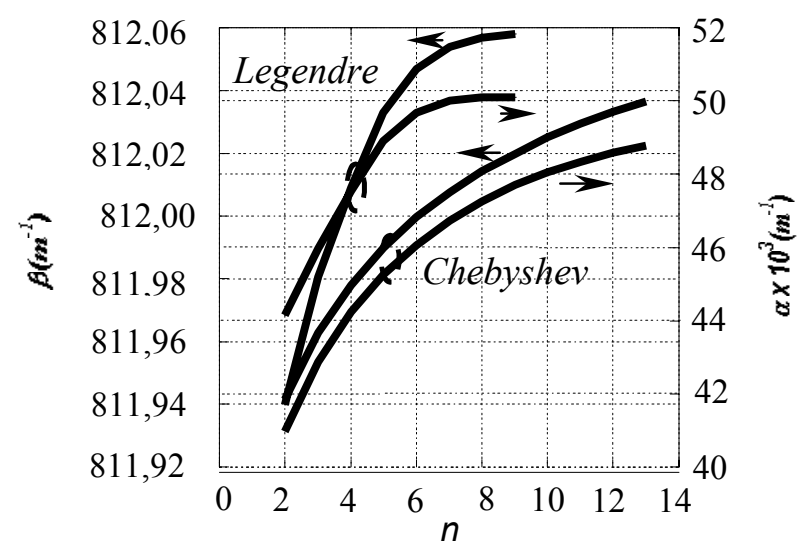

Fig. 3. Propagation constant $\beta$ and attenuation coefficient $\alpha$ versus number of basis functions for an YBCO (The London penetration depth $\lambda_{L}=0.33 \mu \mathrm{m}$, the normal conductivity $\sigma_{n}=$ $\left.3.0 \cdot 10^{6}(\mathrm{Ohm} \cdot \mathrm{m})^{-1}\right)$ microstrip line on $\mathrm{LaAlO}_{3}$ substrate $(\mathrm{f}=$ $10 \mathrm{GHz}$ ). The line with $w=180 \mu \mathrm{m}$ and $t=0.4 \mu \mathrm{m}$ was placed on the substrate with $h_{\text {Low }}^{l}=0.508 \mathrm{~mm}, \varepsilon=23.5$ and $\tan \delta=6.0 \cdot 10^{8}$.

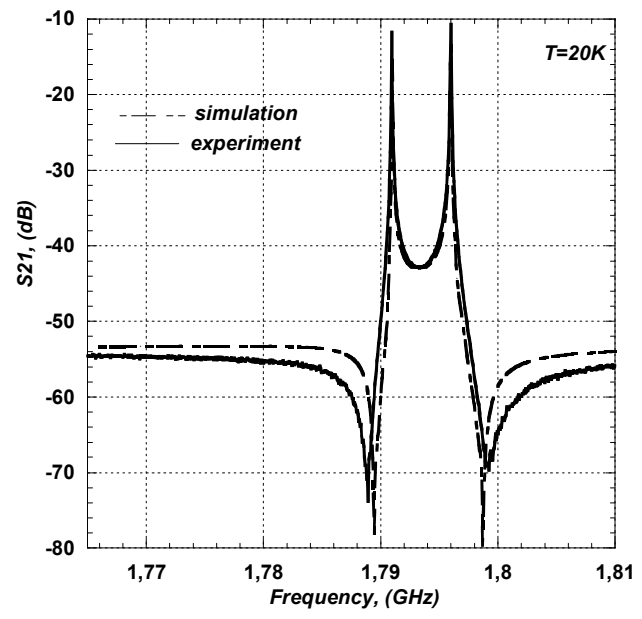

a)

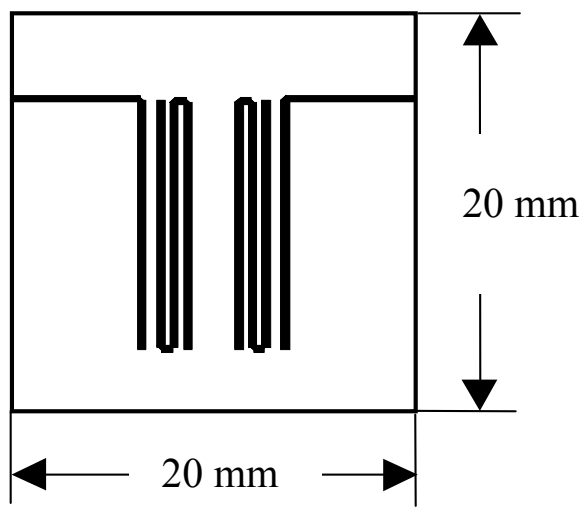

Fig. 4. The layout of two S-shape YBCO resonators on $\mathrm{LaAlO}_{3}$ substrate. The copper $\left(\sigma=7 \cdot 10^{7}\right.$ $\left.(\mathrm{Ohm} \cdot \mathrm{m})^{-1}\right)$ box dimensions are $L=25 \mathrm{~mm}$ and $\mathrm{h}_{\mathrm{Up}}^{1}=7 \mathrm{~mm}$.

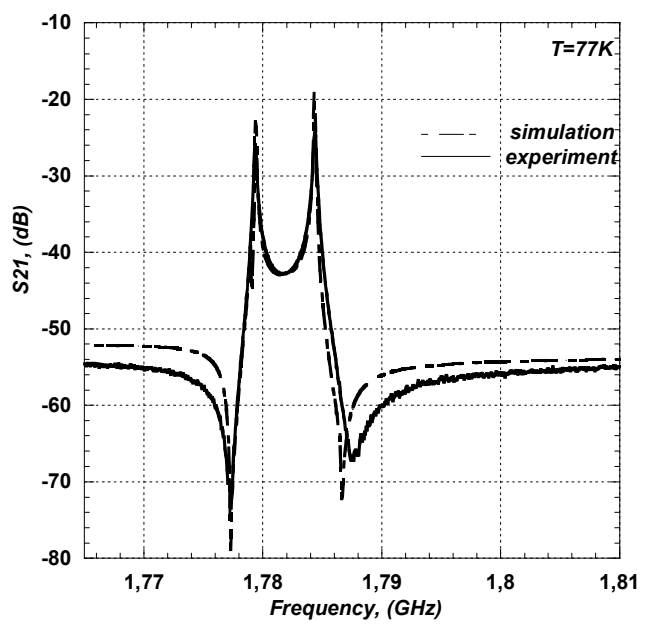

b)

Fig. 5. The simulated and measured Vendik et al. (6) transmission characteristics of two coupled S-shape resonators (Fig. 4). The dielectric substrate of $0.515 \mathrm{~mm}$ thickness was used with parameters $\varepsilon=23.3$, $\tan \delta=2.0 \cdot 10^{-6}$, the YBCO film of $0.45 \mu \mathrm{m}$ thickness was used with characteristics $\lambda_{L}=0.144 \mu \mathrm{m}, \sigma_{n}=5.25 \cdot 10^{6}(\mathrm{Ohm} \cdot \mathrm{m})^{-1}$ (a) and $\lambda_{L}=0.282$ $\mu \mathrm{m}, \sigma_{n}=12.4 \cdot 10^{6}(\mathrm{Ohm} \cdot \mathrm{m})^{-1}(\mathrm{~b})$.

\section{REFERENCES}

1. I. B. Vendik, O. G. Vendik, A. N. Deleniv, V. V. Kondratiev, M. N. Goubina, and D. V. Kholodniak, "Development of CAD tool for a design of microwave planar HTS filters", 2000, IEEE Trans. Microwave Theory Tech., vol. 48, pp. 12471255.

2. I. Vendik, "Phenomenological model of the microwave surface impedance of high-Tc superconducting films", 2000, Supercond. Sci. Technol., vol. 13, pp. 974-982.

3. T. Itoh, "Spectral domain immitance approach for dispersion characteristics of generalized printed transmission lines", 1980, IEEE Trans. Microwave Theory Tech., vol. 28, pp. 733-736.

4. S. Van den Berghe, F. Olyslager, and D. De Zutter, "Accurate modeling of thin conducting layers in FDTD", 1998, IEEE Microwave and Guided Wave Letters, vol. 8, pp. 75-77.

5. G. Cano, F. Medina, and M. Horno, On the efficient Implementation of SDA for boxed strip-like and slot-like structures", 1998, IEEE Trans. Microwave Theory Tech., vol. 46, pp. 1801-1806.

6. I. B. Vendik, A. N. Deleniv, V. O. Sherman, A. A. Svishchev, V. V. Kondratiev, D. V. Kholodniak, A. V. Lapshin, P. N. Yudin, B.-C. Min, Y. H. Choi, and B. Oh, "Narrowband Y-Ba-Cu-O filter with quasi-elliptic characteristic", 2001, Appl. Supercond., vol. 11, pp. 477-480. 